\title{
Natural Convection in a Trapezoidal Enclosure with Wavy Top Surface
}

\author{
Pensiri Sompong ${ }^{1,2}$ and Supot Witayangkurn ${ }^{1}$ \\ ${ }^{1}$ Department of Mathematics, Faculty of Science, Khon Kaen University, Khon Kaen 40002, Thailand \\ ${ }^{2}$ Centre of Excellence in Mathematics, CHE, Si Ayutthaya Road, Bangkok 10400, Thailand \\ Correspondence should be addressed to Supot Witayangkurn; supot_wa@kku.ac.th
}

Received 15 May 2013; Accepted 8 October 2013

Academic Editor: Subhas Abel

Copyright (c) 2013 P. Sompong and S. Witayangkurn. This is an open access article distributed under the Creative Commons Attribution License, which permits unrestricted use, distribution, and reproduction in any medium, provided the original work is properly cited.

\begin{abstract}
The effects of various parameters, Rayleigh number (Ra), Darcy number (Da), and wave amplitude (a), on natural convection inside a trapezoidal enclosure with wavy top surface are studied. The enclosure is filled with seawater having Prandtl number (Pr) of 7.2 and uniformly heated on bottom and partially heated on inclined boundaries. The flow field and temperature distribution are observed when interested parameters are chosen for $\mathrm{Ra}=10^{4}, 10^{5}$, and $10^{6}, \mathrm{Da}=10^{-5}, 10^{-4}$, and $10^{-3}$, and $a=0.9,1$, and 1.1. FlexPDE, a finite element model builder, is used to solve the governing equations to obtain the numerical results displayed by streamlines and isotherms. From the study results, convection motion is affected by different parameters in which the increase in flow intensity and temperature distribution can be seen at higher Rayleigh and Darcy numbers. The wavy top surface has small influence on the flow field and temperature distribution compared to the influence of Rayleigh and Darcy numbers.
\end{abstract}

\section{Introduction}

The study of natural convection in an enclosure has received significant attention because the convection motion plays an important role in thermal characteristic and can be found in many applications. Due to a large number of technical applications, for example, geophysics, geothermal reservoirs, insulation of building, crude oil production, separation processes in industries, and so on, many researchers have studied natural convection inside the different shapes of enclosures, triangle, rectangle, nonrectangle, or irregular enclosure like wavy surface, with various boundary conditions to analyze the fluid flow and thermal behaviors. Triangular enclosure can be used in the application of roof structure in order to study the natural convection under summer and winter day boundary conditions which are found in Asan and Namli $[1,2]$. Koca et al. [3] also analyzed the fluid flow in triangular enclosure with localized heating in which bottom wall is heated partially, while the inclined wall is cooled. Literature reviews on natural convection inside the square and rectangular enclosure having different temperature boundary condition, and filled with porous medium are available in Baytas and Pop [4], Corcione [5], Basak et al. [6], Sathiyamoorthy et al. [7], and Khansila and Witayangkurn [8]. For nonrectangular enclosure filled with porous medium, Baytaş and Pop [9] investigated the steady natural convection within an inclined trapezoidal enclosure that the top and bottom cylindrical surfaces are cooled and heated, respectively. Varol et al. [10] presented a numerical study of natural convection in a right-angle trapezoidal cavity which is heated on the left vertical wall and partially cooled from the inclined wall whereas a finite element simulation of natural convection flow in a trapezoidal enclosure which is uniformly and nonuniformly heated from below is studied by Basak et al. [11].

For a complicated enclosure with wavy surface, Das and Mahmud [12] investigated the natural convection inside the enclosure which consists of two isothermal wavy walls (top and bottom) and two adiabatic straight walls (left and right). Dalal and Das $[13,14]$ were interested in a square enclosure having three flat walls and wavy right vertical walls consisting of one, two, and three undulations. With the same model, numerical study adding heat function equation to study heat 
transport for convection is presented in Dalal and Das [15]. In addition, Oztop et al. [16] considered the enclosure that the vertical walls are heated differentially whereas two wavy walls (top and bottom) are adiabatic. Sompong and Witayangkurn [17] studied the natural convection in a square enclosure having two wavy vertical walls. The enclosure is heated spatially on bottom wall, while remaining walls are maintained at lower temperature. Hasan et al. [18] investigated the effects of corrugation frequency and aspect ratio on natural convection in an enclosure with top wavy surface motivated by a summer day house exposed to sunlight on the roof.

The objective of this study is to investigate the flow field and temperature distribution due to natural convection inside the trapezoidal enclosure with wavy top surface where the enclosure is heated from bottom and discrete heat sources are located on inclined boundaries by using finite element method. The physical model and mathematical formulation are described in Section 2. For Sections 3 and 4, the computational results and conclusion are discussed.

\section{Physical Model and Mathematical Formulation}

The following notations are applied throughout the paper.

$a, b$ : Contraction ratio of wavy wall

$g$ : Acceleration due to gravity $\left(\mathrm{m} \mathrm{s}^{-2}\right)$

$L$ : Base length or height of enclosure $(\mathrm{m})$

$p$ : Pressure $(\mathrm{Pa})$

$T$ : Temperature (K)

$T_{C}$ : Temperature of cold wall $(\mathrm{K})$

$T_{H}$ : Temperature of hot wall $(\mathrm{K})$

$v$ : Kinematic viscosity $\left(\mathrm{m}^{2} \mathrm{~s}^{-1}\right)$

$\rho:$ Density $\left(\mathrm{kg} \mathrm{m}^{-3}\right)$

$x, y$ : Cartesian coordinates $(\mathrm{m})$

$u, v: x, y$ components of velocity $\left(\mathrm{m} \mathrm{s}^{-1}\right)$

Da: Darcy number

$K$ : Permeability of the porous medium

$P$ : Dimensionless pressure

Pr: Prandtl number

Ra: Rayleigh number

$\gamma$ : Penalty parameter

$\theta$ : Dimensionless temperature

$\psi:$ Stream function

$X, Y$ : Dimensionless distance along $x, y$ coordinate

$U, V: x, y$ components of dimensionless velocity.

Figure 1 shows physical model of a trapezoidal enclosure with wavy top boundary in which the top boundary is described by (1), where $b=1-a$, and $L$ indicates the base length and height of the enclosure. The left and right boundaries are inclined by angle $\phi=45^{\circ}$ and heat sources with size $\delta \sqrt{2}$, where $\delta=\varepsilon L$, are located on them. Consider

$$
f(x)=L\left(a+b \cos \left(\frac{2 \pi x}{L}\right)\right) .
$$

For the boundary conditions, it is assumed that there is no slip on boundary. Thus, the velocities in $x$ and $y$ directions are $u=0$ and $v=0$ for all boundaries. The bottom boundary is uniformly heated with temperature $T=$ $T_{H}$, while the inclined boundaries are linearly heated. The remaining boundaries are cooled with temperature $T=T_{C}$. Natural convection can be described by differential equations of conservation of mass, momentum, and energy (see [6]). By using the following change of variables

$$
\begin{gathered}
X=\frac{x}{L}, \quad Y=\frac{y}{L}, \quad U=\frac{u L}{\alpha}, \quad V=\frac{v L}{\alpha}, \\
\theta=\frac{T-T_{C}}{T_{H}-T_{C}}, \quad P=\frac{p L^{2}}{\rho \alpha^{2}}, \quad \operatorname{Pr}=\frac{\nu}{\alpha}, \\
\mathrm{Ra}=\frac{g \beta\left(T_{H}-T_{C}\right) L^{3} \operatorname{Pr}}{\nu^{2}}, \quad \mathrm{Da}=\frac{K}{L^{2}}
\end{gathered}
$$

the equations for steady two-dimensional natural convection flow in the porous cavity in term of dimensionless are

$$
\begin{gathered}
\frac{\partial U}{\partial X}+\frac{\partial V}{\partial Y}=0 \\
U \frac{\partial U}{\partial X}+V \frac{\partial U}{\partial Y}=-\frac{\partial P}{\partial X}+\operatorname{Pr}\left(\frac{\partial^{2} U}{\partial X^{2}}+\frac{\partial^{2} U}{\partial Y^{2}}\right)-\frac{\operatorname{Pr}}{\mathrm{Da}} U \\
U \frac{\partial V}{\partial X}+V \frac{\partial V}{\partial Y}=-\frac{\partial P}{\partial Y}+\operatorname{Pr}\left(\frac{\partial^{2} V}{\partial X^{2}}+\frac{\partial^{2} V}{\partial Y^{2}}\right) \\
-\frac{\operatorname{Pr}}{\operatorname{Da}}+\operatorname{RaPr} \theta \\
U \frac{\partial \theta}{\partial X}+V \frac{\partial \theta}{\partial Y}=\frac{\partial^{2} \theta}{\partial X^{2}}+\frac{\partial^{2} \theta}{\partial Y^{2}}
\end{gathered}
$$

The transformed boundary conditions are changed to $U=0$ and $V=0$ for all boundaries and $\theta=1$ for the bottom. The temperatures of the inclined boundaries are as follows: on the left,

$$
\theta= \begin{cases}1-Y, & \text { for } \frac{1}{2}(\varepsilon-1)-\varepsilon \leq X \leq \frac{1}{2}(\varepsilon-1), \\ 0, & \text { otherwise }\end{cases}
$$

on the right,

$$
\theta= \begin{cases}1-Y, & \text { for } \frac{1}{2}(3-\varepsilon) \leq X \leq \frac{1}{2}(3-\varepsilon)+\varepsilon, \\ 0, & \text { otherwise. }\end{cases}
$$

The temperature of the rest of the boundaries is $\theta=$ 0 . By using the penalty finite element method with 


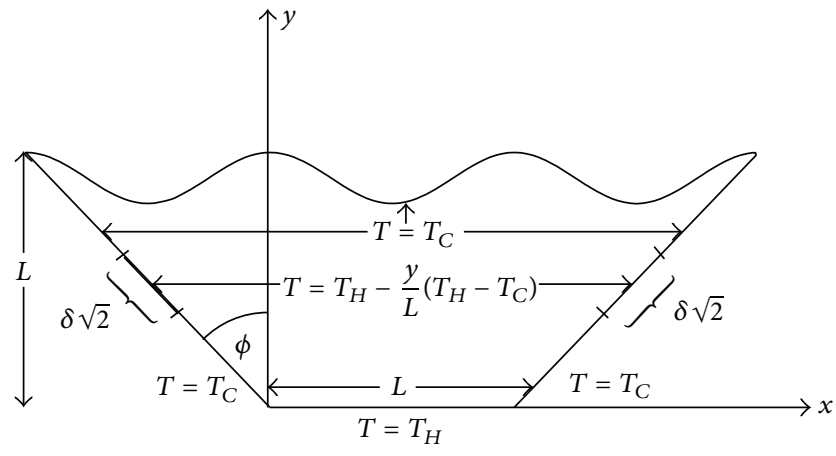

FIGURE 1: Physical model and boundary conditions of the enclosure with top wavy surface.

$P=-\gamma(\partial U / \partial X+\partial V / \partial Y)$, where $\gamma=10^{7}$ [19], and the relationships between stream function [20], $U=\partial \psi / \partial Y$ and $V=-\partial \psi / \partial X,(3)$ are expressed by

$$
\begin{gathered}
\frac{\partial^{2} \psi}{\partial X^{2}}+\frac{\partial^{2} \psi}{\partial Y^{2}}=\frac{\partial U}{\partial Y}-\frac{\partial V}{\partial X} \\
U \frac{\partial U}{\partial X}+V \frac{\partial U}{\partial Y}= \\
\gamma \frac{\partial}{\partial X}\left(\frac{\partial U}{\partial X}+\frac{\partial V}{\partial Y}\right) \\
+\operatorname{Pr}\left(\frac{\partial^{2} U}{\partial X^{2}}+\frac{\partial^{2} U}{\partial Y^{2}}\right)-\frac{\operatorname{Pr}}{\mathrm{Da}} U \\
U \frac{\partial V}{\partial X}+V \frac{\partial V}{\partial Y}=\gamma \frac{\partial}{\partial Y}\left(\frac{\partial U}{\partial X}+\frac{\partial V}{\partial Y}\right) \\
+\operatorname{Pr}\left(\frac{\partial^{2} V}{\partial X^{2}}+\frac{\partial^{2} V}{\partial Y^{2}}\right)-\frac{\operatorname{Pr}}{\mathrm{Da}} V+\operatorname{RaPr} \theta
\end{gathered}
$$

The top wavy surface shown in (1) is rewritten as

$$
f(X)=a+b \cos (2 \pi X) .
$$

With the physical model and boundary conditions mentioned above, the fluid saturated porous media considered incompressible and Newtonian are contained inside a cavity and its properties are constant except the density variation. This study is performed on different parameters in which Rayleigh number $(\mathrm{Ra})$ is ranging from $10^{4}$ to $10^{6}$, Darcy number is ranging from $10^{-5}$ to $10^{-3}$, and wave amplitude is ranging from 0.9 to 1.1. Here, Prandtl number (Pr) is kept constant at 7.2 (in the case of seawater). In addition, the effects on flow field and temperature distribution are analyzed.

\section{Results and Discussion}

To analyze the effects on the flow and temperature characteristic, the governing equations mentioned previously are coded into FlexPDE which is a software package performing the operations necessary to turn a description of a partial differential equations system into a finite element model.
Then, it solves the system and presents graphical and tabular outputs of the results. The results obtained from varying values of interested parameters are displayed by streamlines and isotherms in which graphical outputs from FlexPDE are modified to show numerical values.

Figures 2 and 3 illustrate the effects of different parameters on the flow field and temperature distribution. Figure 2 shows the results for different $\mathrm{Ra}\left(\mathrm{Ra}=10^{4}, 10^{5}\right.$, and $10^{6}$ ) with $\mathrm{Da}=10^{-3}$ and $a=0.9$. It can be seen that two main circulations rotating in different direction occur inside a cavity. As seen from value of stream function, the left half is positive value and the right half is negative indicating anticlockwise and clockwise circulation patterns, respectively. Because the enclosure is heated from below, the fluid moves upwards and impings to the top of the enclosure and then flows down along the side boundaries forming two symmetric circulations. The flow field near the side walls is little distorted because two heat sources are located on inclined boundaries. It is noted that there is no circulation at the top corner because of no temperature difference. The flow intensity increases with increasing $\mathrm{Ra}$. At low $\mathrm{Ra}\left(\mathrm{Ra}=10^{4}\right)$, maximum value is $\left|\psi_{\max }\right|=0.25$ as seen from Figure 2(a). It is observed that circulation center is near bottom corner and magnitude of streamlines is small due to the influence of conduction. As $\mathrm{Ra}$ is increased to $10^{5}$, maximum value is $\left|\psi_{\max }\right|=3.5$ and circulation center moves up near the middle center line (Figure 2(b)), while the intensity of flow increases to $\left|\psi_{\max }\right|=12$ at the highest Ra (Figure 2(c)) because fluid motion is driven by the influence of convection resulting in larger cell center.

The effects of increasing $\mathrm{Ra}$ on temperature distribution are illustrated by isotherms as shown in the second column of Figure 2. Because the enclosure is heated on the bottom and partially heated on inclined boundaries, isothermal stratifications occur near heated region which indicates the temperature gradient. The temperature line $\theta=0.1$ is near the wavy top boundary and it is the same level as streamlines values $|\psi|=0.05$ for $\mathrm{Ra}=10^{4}$. It is observed that the temperature distribution is smooth curve and stationary for low $\mathrm{Ra}$ because the fluid flow is very weak as seen from streamline value, while the isotherm pattern changes significantly for higher $\mathrm{Ra}$ indicating that the convection is the principle mode. For $\mathrm{Ra}=10^{5}$, isothermal lines are pushed towards the upper portion. The temperatures $\theta=0.1$ and 0.2 are wavy like the top boundary and the distortion of temperature $\theta \geq 0.3$ slightly increases because of stronger circulation. The temperature contours are compressed at the upper and lower portion and more compressed near the bottom when $\mathrm{Ra}$ is increased to $\mathrm{Ra}=10^{6}$ due to the enhancement of flow circulation inside the enclosure as seen from larger magnitude of stream function.

Streamlines and isotherms for different $\mathrm{Da}$ with constant $\mathrm{Ra}=10^{6}$ are shown in Figure 3. It can be seen that the fluid motion is very weak for $\mathrm{Da}=10^{-5}$ and the shapes of streamlines and isotherms are similar to the previous case for $\mathrm{Ra}=10^{4}$ with constant $\mathrm{Da}=10^{-3}$ as shown in Figure 2(a). For $\mathrm{Ra}=10^{6}$ with $\mathrm{Da}=10^{-4}$ (Figure 3(b)), fluid circulation is stronger and isotherm pattern expands the 

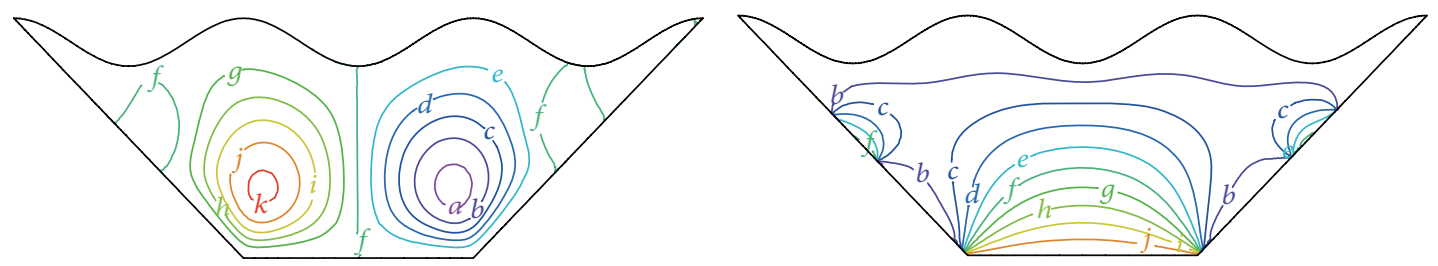

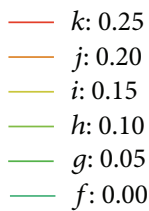

$$
\begin{gathered}
e:-0.05 \\
d:-0.10 \\
c:-0.15 \\
b:-0.20 \\
a:-0.25
\end{gathered}
$$
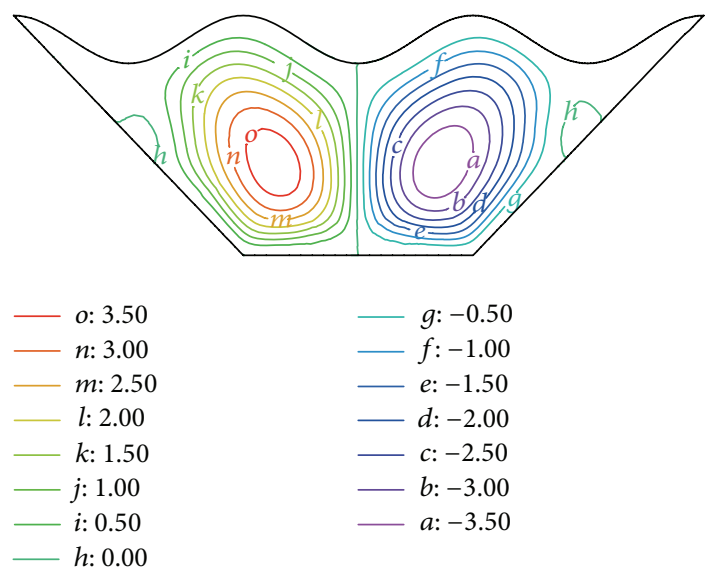

$$
\begin{aligned}
& \text { - } g:-0.50 \\
& \text { - } f:-1.00 \\
& \text { - e: }-1.50 \\
& \text { - } d:-2.00 \\
& c:-2.50 \\
& \text { - } b:-3.00 \\
& a:-3.50
\end{aligned}
$$

(b)

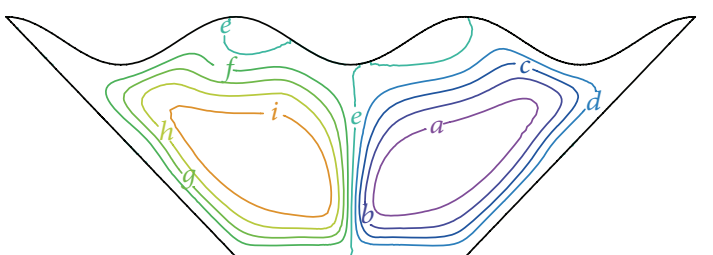

(a)

)

$$
\begin{aligned}
& \text { - } d:-3.00 \\
& c:-6.00 \\
& \text { - } b:-9.00 \\
& \text { - } a:-12.0
\end{aligned}
$$

$f: 3.00$ $e: 0.00$

Figure 2: Streamlines (left) and isotherms (right) for $\mathrm{Da}=10^{-3}$ and $a=0.9$. (a) $\mathrm{Ra}=10^{4}$, (b) $\mathrm{Ra}=10^{5}$, and (c) $\mathrm{Ra}=10^{6}$.

entire enclosure compared to the case of $\mathrm{Da}=10^{-5}$. The influence of convection resulting in stronger fluid intensity and larger temperature distribution can be observed when $\mathrm{Da}$ is increased to $10^{-3}$. In addition, fluid circulation is stronger and temperature distribution becomes larger when $\mathrm{Ra}$ is increased with constant $\mathrm{Da}$ or $\mathrm{Da}$ is increased with constant $\mathrm{Ra}$. Note that the intensity of flow circulation and temperature distribution depend on $\mathrm{Ra}$ and $\mathrm{Da}$ in which the range of these values has an important role to define the conduction and convection dominant.
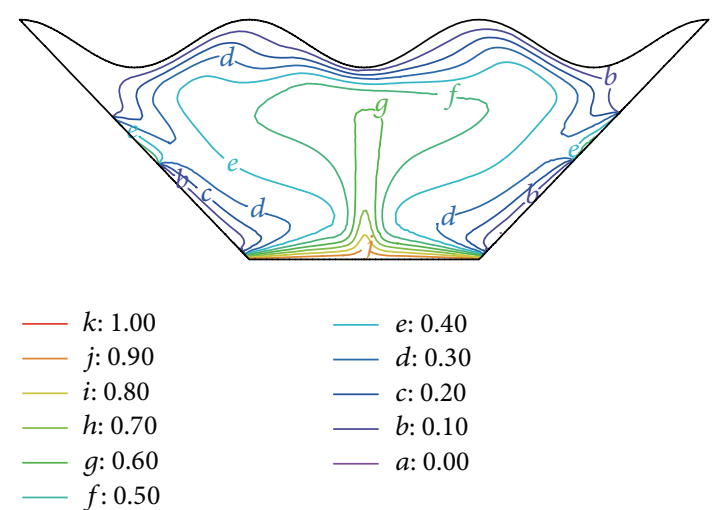

(c)
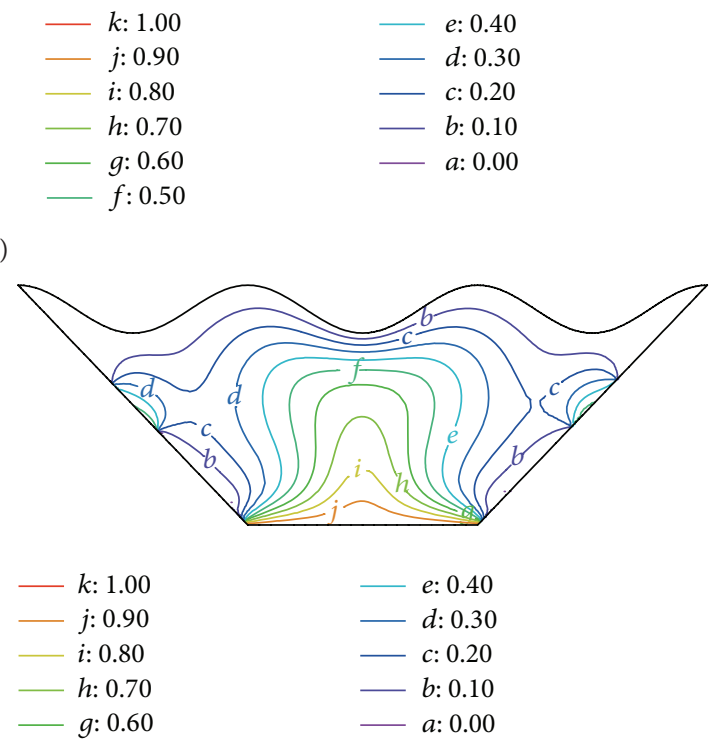

$f: 0.50$

c)

The results obtained from changing wave amplitude are shown in Figure 4 . The wavy top surface does not have an effect on flow intensity as seen from the maximum value of streamlines, $\left|\psi_{\max }\right|=4$, for all values of wave amplitude. The shape of flow circulation is almost elliptic and fits with the wavy surface of the enclosure. The increase of $a$ from 0.9 to 1 results in less compression for temperature $\theta \leq 0.6$ because of higher wave volume. For $\mathrm{Ra}=10^{6}$ with $\mathrm{Da}=10^{-4}$, the buoyancy force generated is strong enough to initiate fluid convection. Fluid rises up from middle portion of bottom 

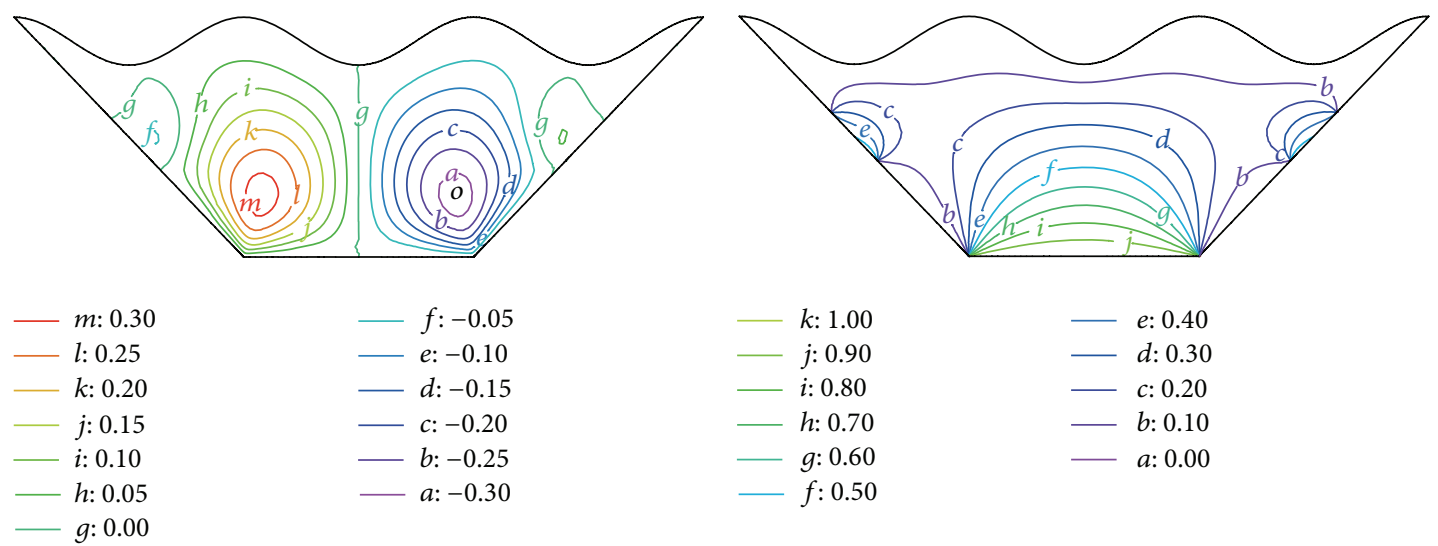

(a)
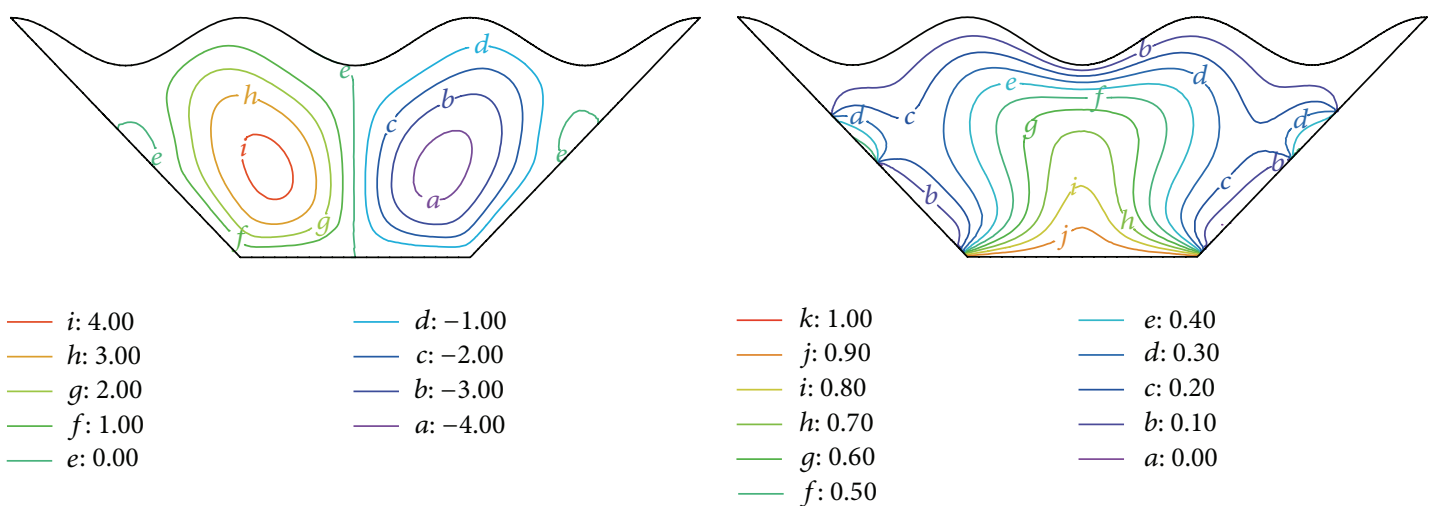

(b)
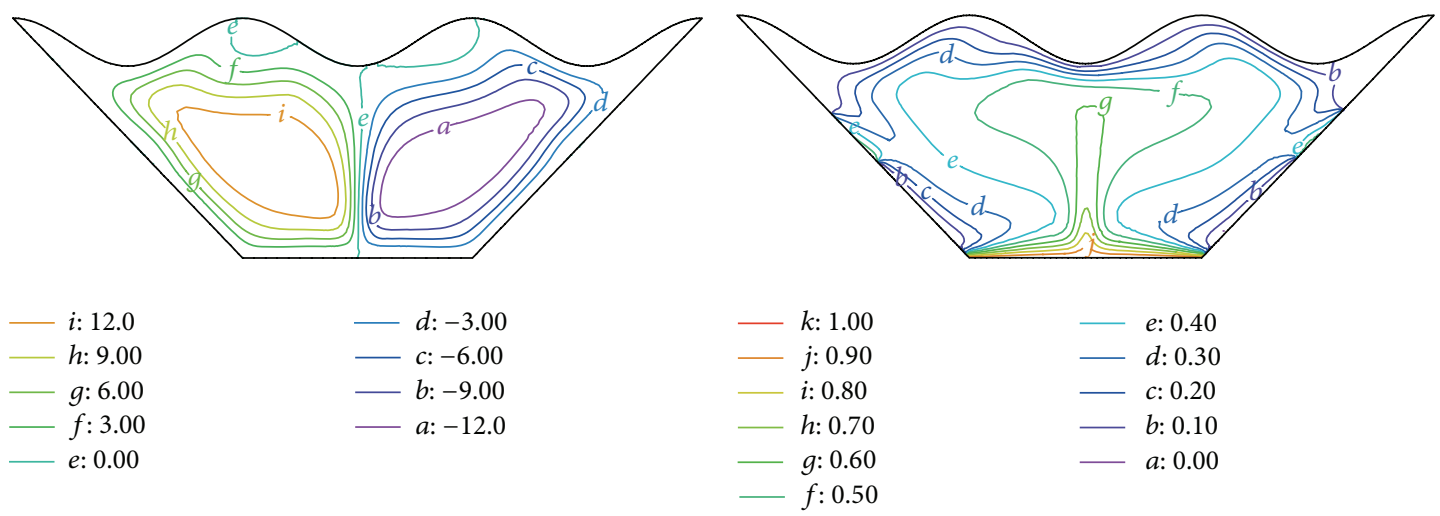

(c)

Figure 3: Streamlines (left) and isotherms (right) for $a=0.9$ and $\mathrm{Ra}=10^{6}$. (a) $\mathrm{Da}=10^{-5}$, (b) $\mathrm{Da}=10^{-4}$, and (c) $\mathrm{Da}=10^{-3}$.

towards the top surface causing the outermost streamlines to be distorted by the inner wave for $a=1$. It can be seen that isotherms are pushed towards upper portion of the enclosure and distributed on the entire enclosure except the top corner region. As $a$ is increased to 1.1, the wave volume is higher and temperature distribution fits with the middle wave.

\section{Conclusion}

The effects of interested parameters, Rayleigh number, Darcy number, and wave amplitude on natural convection inside the trapezoidal enclosure with wavy top boundary are investigated. The values of parameters have been chosen based on wide range of applicability, $\mathrm{Ra}=10^{4}, 10^{5}$, and $10^{6}, \mathrm{Da}=10^{-5}$, $10^{-4}$, and $10^{-3}$, and $a=0.9,1$, and 1.1 with constant $\operatorname{Pr}=7.2$. Interesting results are obtained and displayed by streamlines and isotherms. From the study results, it is found that

(i) the magnitudes of the flow and temperature distribution are small for $\mathrm{Ra}=10^{4}$ and $\mathrm{Da}=10^{-3}$ due to the influence of conduction, while the increase of $\mathrm{Ra}$ $\left(\mathrm{Ra}=10^{5}\right.$ and $\left.10^{6}\right)$ enhances the strength of fluid 

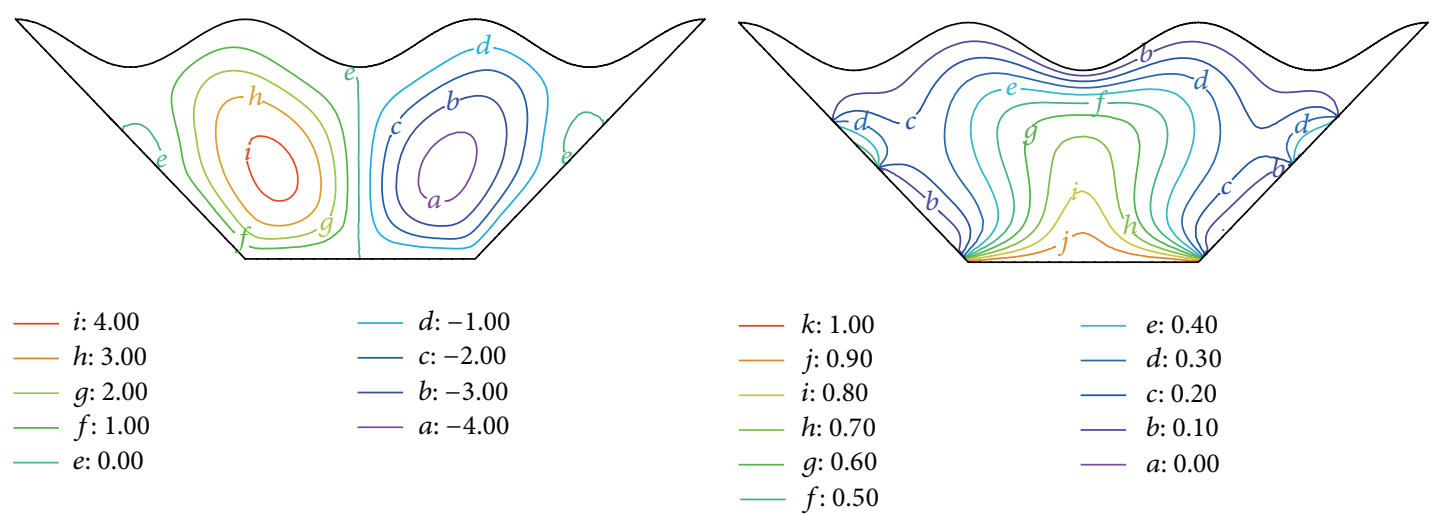

(a)
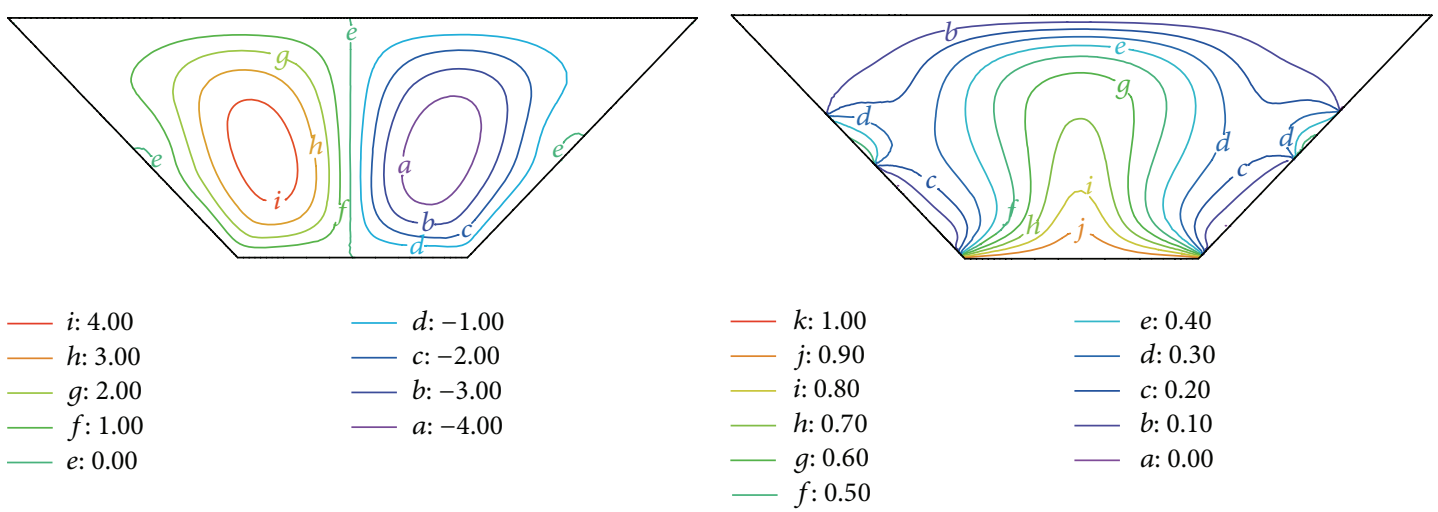

(b)
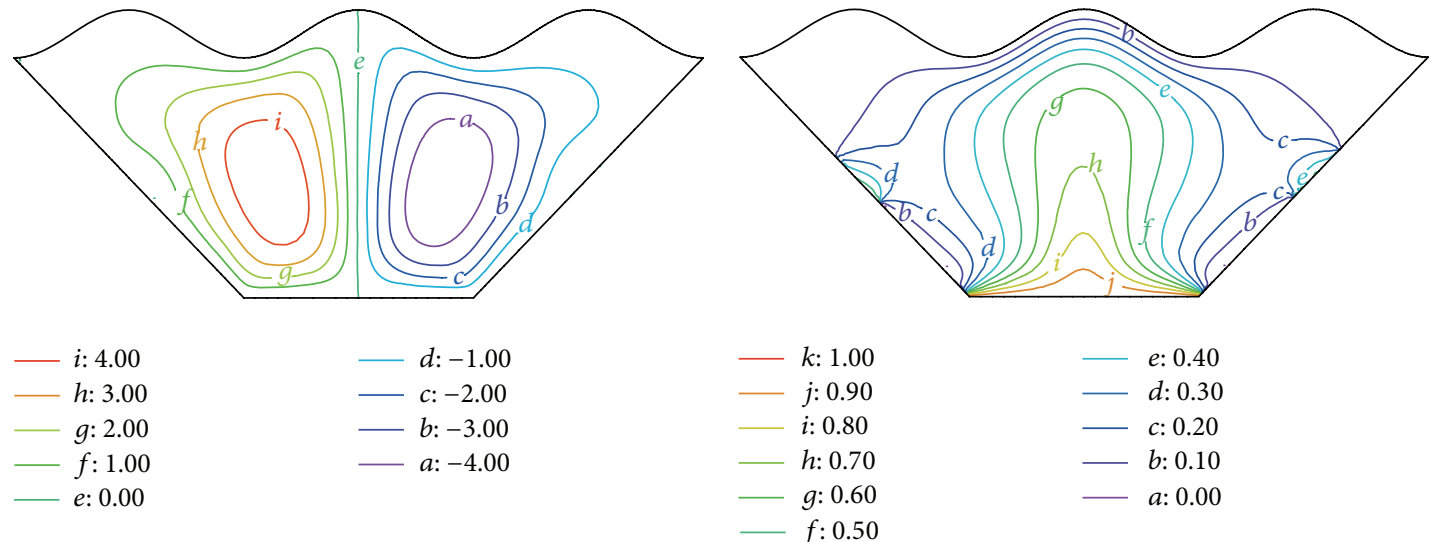

(c)

Figure 4: Streamlines (left) and isotherms (right) for $\mathrm{Ra}=10^{6}$ and $\mathrm{Da}=10^{-4}$. (a) $a=0.9$, (b) $a=1$, and (c) $a=1.1$.

flow and temperature distribution indicating a role of convection dominant,

(ii) at $\mathrm{Da}=10^{-5}$ and $\mathrm{Ra}=10^{6}$, the flow intensity is very weak resulting in less temperature distribution. The intensity of circulation is stronger and temperature gradient is larger for higher $\mathrm{Da}\left(\mathrm{Da}=10^{-4}\right.$ and $\left.10^{-3}\right)$,

(iii) the result of a variation in $\mathrm{Ra}$ shows similar trend with a variation in $\mathrm{Da}$; that is, the fluid circulation at constant $\mathrm{Da}\left(\mathrm{Da}=10^{-3}\right)$ is stronger as $\mathrm{Ra}$ is increased and the fluid circulation at constant $\mathrm{Ra}\left(\mathrm{Ra}=10^{6}\right)$ is stronger as $\mathrm{Da}$ is increased,

(iv) fluid circulation is not found at the top corners of the enclosure for all cases,

(v) the increase of wave amplitude from 0.9 to 1.1 has no effect on flow intensity but it affects the flow pattern due to the inner wave,

(vi) the wavy top surface has small influence on temperature distribution compared to the influence of $\mathrm{Ra}$ and Da. 


\section{Acknowledgments}

This research is (partially) supported by Centre of Excellence in Mathematics, the Commission on Higher Education, Thailand. The authors would like to thank Department of Mathematics, Faculty of Science, Khon Kaen University (Thailand), for computational resources necessary for this work.

\section{References}

[1] H. Asan and L. Namli, "Laminar natural convection in a pitched roof of triangular cross-section: summer day boundary conditions," Energy and Buildings, vol. 33, no. 1, pp. 69-73, 2000.

[2] H. Asan and L. Namli, "Numerical simulation of buoyant flow in a roof of triangular cross-section under winter day boundary conditions," Energy and Buildings, vol. 33, no. 7, pp. 753-757, 2001.

[3] A. Koca, H. F. Oztop, and Y. Varol, "The effects of Prandtl number on natural convection in triangular enclosures with localized heating from below," International Communications in Heat and Mass Transfer, vol. 34, no. 4, pp. 511-519, 2007.

[4] A. C. Baytas and I. Pop, "Free convection in a square porous cavity using a thermal nonequilibrium model," International Journal of Thermal Sciences, vol. 41, no. 9, pp. 861-870, 2002.

[5] M. Corcione, "Effects of the thermal boundary conditions at the sidewalls upon natural convection in rectangular enclosures heated from below and cooled from above," International Journal of Thermal Sciences, vol. 42, no. 2, pp. 199-208, 2003.

[6] T. Basak, S. Roy, T. Paul, and I. Pop, "Natural convection in a square cavity filled with a porous medium: effects of various thermal boundary conditions," International Journal of Heat and Mass Transfer, vol. 49, no. 7-8, pp. 1430-1441, 2006.

[7] M. Sathiyamoorthy, T. Basak, S. Roy, and I. Pop, "Steady natural convection flow in a square cavity filled with a porous medium for linearly heated side wall(s)," International Journal of Heat and Mass Transfer, vol. 50, no. 9-10, pp. 1892-1901, 2007.

[8] P. Khansila and S. Witayangkurn, "Visualization of natural convection in enclosure filled with porous medium by sinusoidally temperature on the one side," Applied Mathematical Sciences, vol. 97, pp. 4801-44812, 2012.

[9] A. C. Baytaş and I. Pop, "Natural convection in a trapezoidal enclosure filled with a porous medium," International Journal of Engineering Science, vol. 39, no. 2, pp. 125-134, 2001.

[10] Y. Varol, H. F. Oztop, and I. Pop, "Natural convection in right-angle porous trapezoidal enclosure partially cooled from inclined wall," International Communications in Heat and Mass Transfer, vol. 36, no. 1, pp. 6-15, 2009.

[11] T. Basak, S. Roy, A. Singh, and I. Pop, "Finite element simulation of natural convection flow in a trapezoidal enclosure filled with porous medium due to uniform and non-uniform heating," International Journal of Heat and Mass Transfer, vol. 52, no. 12, pp. 70-78, 2009.

[12] P. K. Das and S. Mahmud, "Numerical investigation of natural convection inside a wavy enclosure," International Journal of Thermal Sciences, vol. 42, no. 4, pp. 397-406, 2003.

[13] A. Dalal and M. K. Das, "Laminar natural convection in an inclined complicated cavity with spatially variable wall temperature," International Journal of Heat and Mass Transfer, vol. 48, no. 18, pp. 3833-3854, 2005.
[14] A. Dalal and M. K. Das, "Natural convection in a cavity with a wavy wall heated from below and uniformly cooled from the top and both sides," Journal of Heat Transfer, vol. 128, no. 7, pp. 717-725, 2006.

[15] A. Dalal and M. K. Das, "Heatline method for the visualization of natural convection in a complicated cavity," International Journal of Heat and Mass Transfer, vol. 51, no. 1-2, pp. 263-272, 2008.

[16] H. F. Oztop, E. Abu-Nada, Y. Varol, and A. Chamkha, "Natural convection in wavy enclosures with volumetric heat sources," International Journal of Thermal Sciences, vol. 50, no. 4, pp. 502514, 2011.

[17] P. Sompong and S. Witayangkurn, "Simulation of natural convection in a complicated enclosure with two wavy vertical walls," Applied Mathematical Sciences, vol. 6, no. 57-60, pp. 2833-2842, 2012.

[18] M. N. Hasan, S. Saha, and S. C. Saha, "Effects of corrugation frequency and aspect ratio on natural convection within an enclosure having sinusoidal corrugation over a heated top surface," International Communications in Heat and Mass Transfer, vol. 39, no. 3, pp. 368-377, 2012.

[19] J. N. Reddy, An Introduction to the Finite Element Method, McGraw-Hill, New York, NY, USA, 1993.

[20] G. K. Batchelor, An Introduction to Fluid Dynamics, Cambridge University Press, Cambridge, Mass, USA, 1993. 


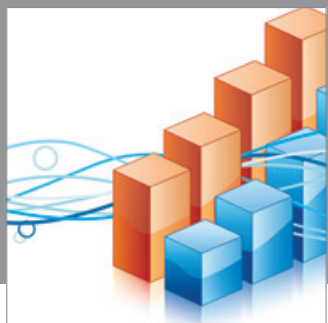

Advances in

Operations Research

mansans

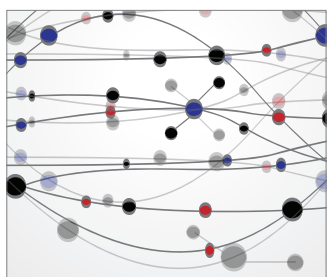

The Scientific World Journal
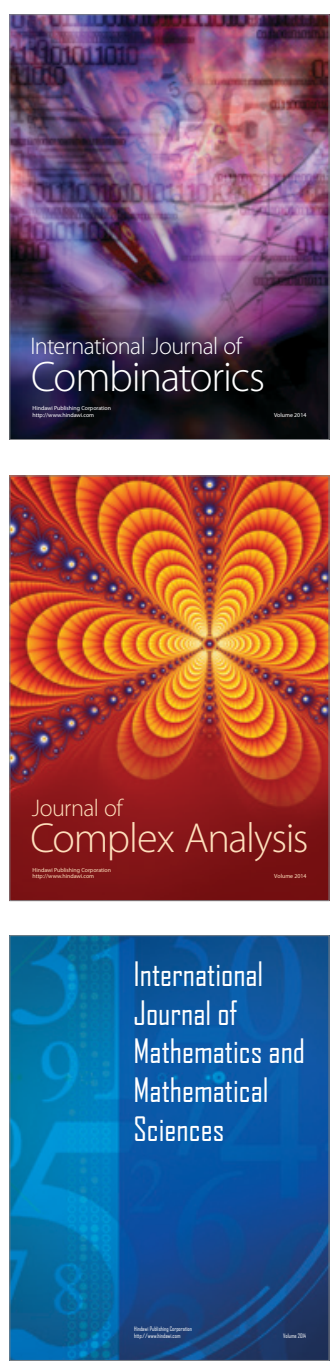
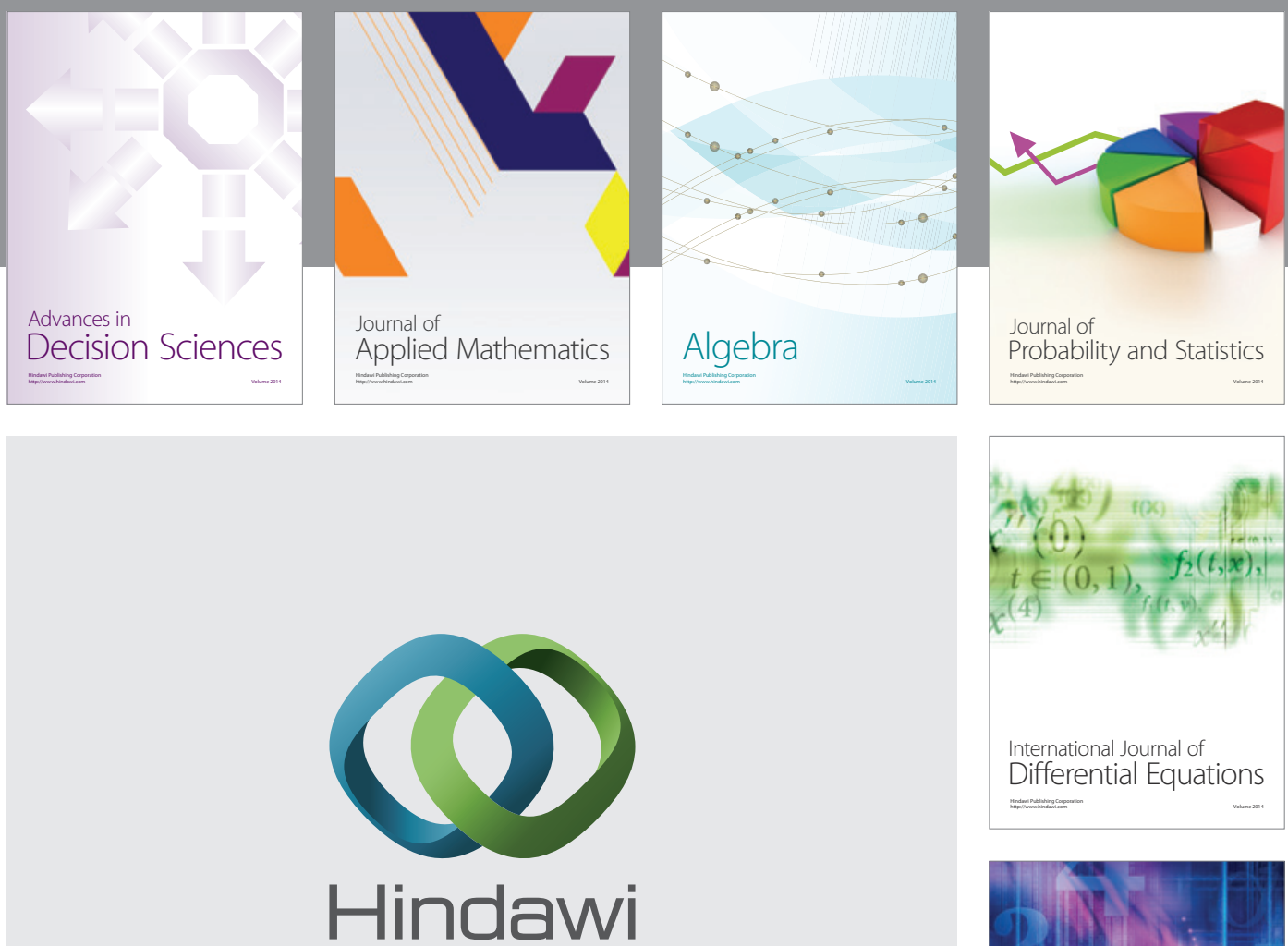

Submit your manuscripts at http://www.hindawi.com
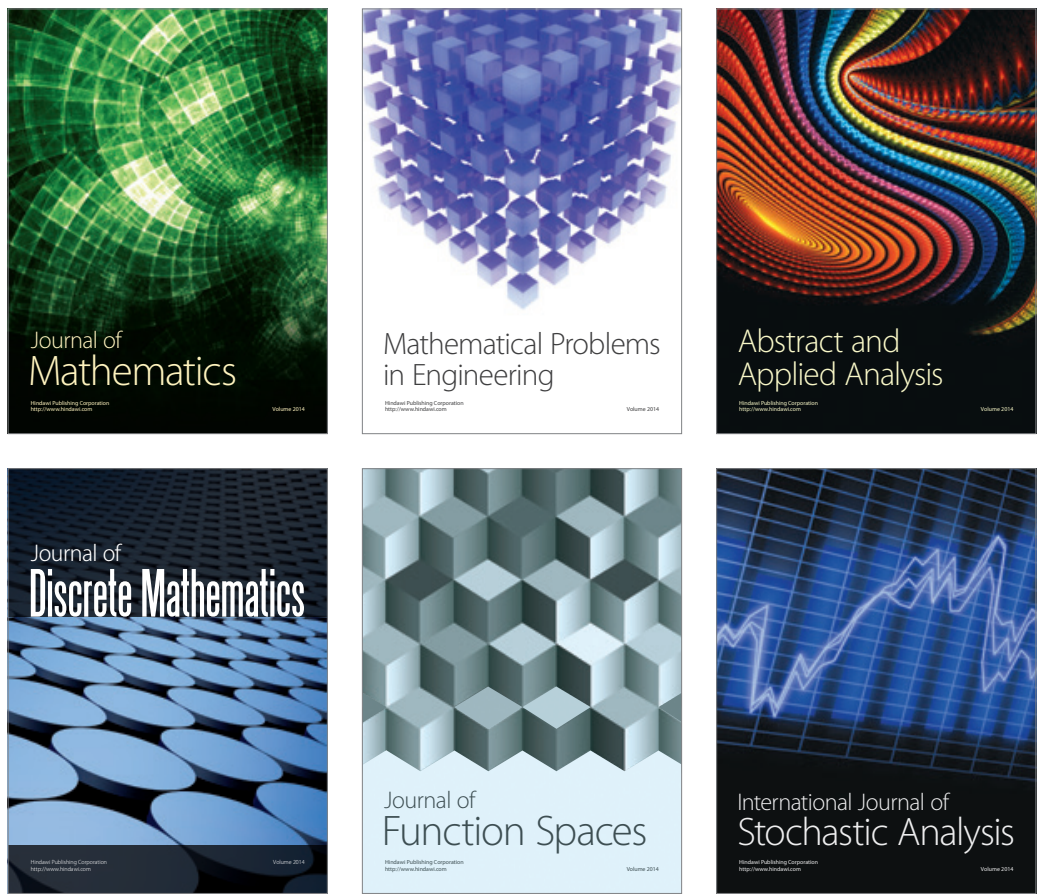

Journal of

Function Spaces

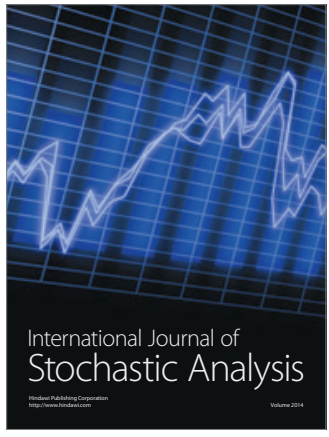

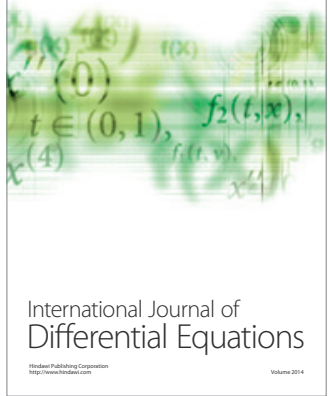
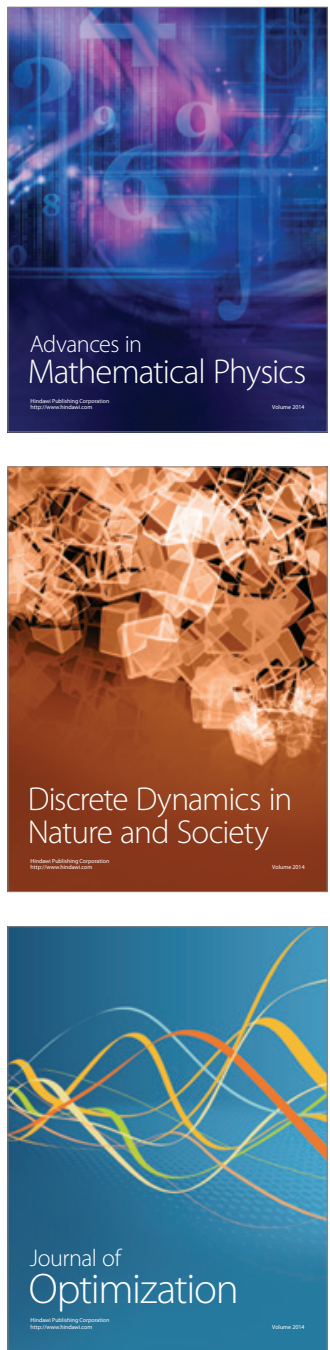